\title{
ON MONOTONE ILL-POSED PROBLEMS IN HILBERT SPACES
}

\author{
NGUYEN BUONG
}

\begin{abstract}
Summary. The main aim of this paper is to study convergence rates for an operator method of regularization to solve nonlinear ill-posed problems involving monotone operators in infinite-dimensional Hilbert space without needing closeness conditions. Then these results are presented in form of combination with finitedimensional approximations of the space. An iterative method for solving regularized equation is given and an example in the theory of singular integral equations is considered for illustration.
\end{abstract}

\section{INTRODUCTION}

Let $H$ be a real Hilbert space with norm and scalar product denoted by $\|$.$\| and \langle.,$.$\rangle ,$ respectively. Let $A$ be a nonlinear operator in $H$ with domain of definition $D(A)=H$ and range $R(A) \subseteq H$, and $f_{0}$ be an element of $R(A)$.

Consider the nonlinear ill-posed problem

$$
A(x)=f_{0}
$$

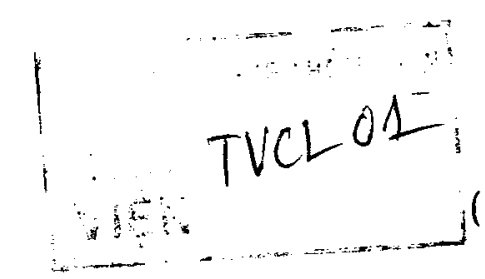

By this we mean that solu ons of (1.1) do not depend continuously on the data $f_{0}$. Various aspects about regularization of (1.1) were studied in detail when $A$ is compact (see, for instance, [6], [13]-[15], [18], [19] and their bibliographies). Here to study convergence rates of variational method of Tikhonov regularization minimizing the functional

$$
F_{\alpha}^{\delta}(x)=\left\|A(x)-f_{\delta}\right\|^{2}+\alpha\|x\|^{2},
$$

where $\alpha>0$ is the parameter of regularization and $f_{\delta}$ are the approximations of $f_{0}$ with the wellknown informations

$$
\left\|f_{\delta}-f_{0}\right\|<\delta, \delta \rightarrow 0,
$$

one needs to have the following conditions (see [6]): (i) $A$ is Fréchet differentiable, (ii) there exists a constant $L>0$ such that $\left\|A^{\prime}(x)-A^{\prime}(y)\right\| \leq L\|x-y\|, x, y \in D(A)$ (iii) there exists an element $\omega \in H$ such that $A^{\prime *}\left(x_{0}\right) \omega=x_{0}$, where $A^{\prime *}\left(x_{0}\right)$ denotes the adjoint of derivative of $A$ at $x_{0}$ and $x_{0}$ is a norm-minimal solution of (1.1), and (iv) $L\|\omega\|<e$ $(=1)$ which is called the closeness condition. In [15] A. Neubauer estimated e $<1$ for a modification of (1.2). 
In [5], when $A$ is a monotone operator, the author obtained $e=2$ for the operator version of Tikhonov regularization

$$
A(x)+\alpha x=f_{\delta},
$$

but convergence rates are a bit weaker. It is clear that the equation in condition (iii) is not defined explicitly because the operator $A^{\prime *}\left(x_{0}\right)$ and the right-hand side $x_{0}$ are not known. Therefore, the verification of (iv) is almost too difficult to realize. So, it is natural to propose the question if there exists a some way excepting condition (iv). In [13] A. Neubauer developed an approach of [11] in the linear case for problems involving compact operators. A big advantage of this approach is that rates are obtained by merely requiring smoothness conditions for the exact solution as in the linear case. In this paper, we shall show that by using a modification of (1.3) that is the regularized equation (see [18])

$$
A(x)+\alpha \partial \varphi(x)=f_{\delta},
$$

where $\partial \varphi$ is the subdifferential of the uniformly convex functional $\varphi$ on $H$, and replacing the smoothness condition (iii) by another one we can exclude condition (iv). Main rèsults about convergence rates are presented in Section 2. An iterative process for (1.4) is given in Section 3 and an numerical example is considered in Section 4 for illustration.

\section{MAIN RESULTS}

Consider the uniformly convex functional $\varphi(x)=\|x\|^{\mu}, 2<\mu<3$. Then (see [7], Lemma 2)

$$
\langle\partial \varphi(x)-\partial \varphi(y), x-y\rangle \geq 2^{2-\mu}\|x-y\|^{\mu}, \forall x, y \in H .
$$

Since $\|x-y\| \geq\|x\|-\|y\|$ it is wellknown in [18] that Eq. (1.4) has a unique solution $x_{\alpha \delta}$ for every fixed $\alpha>0$ and $f_{\delta} \in H$ and the sequence $\left\{x_{\alpha \delta}\right\}$ converges in norm of $H$ to $x_{0}$ if $\delta / \alpha$ and $\alpha$ tend to zero, where

$$
\left\langle\partial \varphi\left(x_{0}\right), x-x_{0}\right\rangle \geq 0, \forall x \in S_{0},
$$

$S_{0}$ is the set of sulutions of (1.1).

We shall prove the following result.

Theorem 2.1. Let the following conditions hold:

(i) $A$ is twice-Fréchet differentiable in some neighbourhood of $x_{0}$, 
(ii) there exists a constant $L>0$ such that

$$
\left\|A^{\prime \prime}(x)-A^{\prime \prime}(z)\right\| \leq L\|x-z\|
$$

for $x, z \in S\left(x_{0}, r\right)$, where $S\left(x_{0}, r\right)$ is a ball with center $x_{0}$ and radius $r$, and

(iii) the equation

$$
\left(A^{\prime *}\left(x_{0}\right)-\frac{1}{2} A^{\prime \prime *}\left(x_{0}\right) y\right) \omega=\partial \varphi\left(x_{0}\right)
$$

has a bounded solution $\omega(y), y \in S\left(x_{0}, r\right)$.

Then for the choice $a=0\left(\delta^{p}\right), 0<p<1$ we obtain

$$
\left\|x_{\alpha \delta}-x_{0}\right\|=0\left(\delta^{q}\right), q=\min ((1-p) /(\mu-1), p / \mu) .
$$

Proof. By virtue of Eqs (1.1) and (1.4) and the monotone property of $A$ we have

$$
\alpha 2^{2-\mu}\left\|x_{\alpha \delta}-x_{0}\right\|^{\mu} \leq \delta\left\|x_{\alpha \delta}-x_{0}\right\|-\alpha\left\langle\partial \varphi\left(x_{0}\right), x_{\alpha \delta}-x_{0}\right\rangle .
$$

From this inequality and condition (iii) of the theorem it follows

$$
\alpha 2^{2-\mu}\left\|x_{\alpha \delta}-x_{0}\right\|^{\mu} \leq \delta\left\|x_{\alpha \delta}-x_{0}\right\|+\alpha\left\langle\omega(y),\left(A^{\prime}\left(x_{0}\right)-\frac{1}{2} A^{\prime \prime}\left(x_{0}\right) y\right)\left(x_{0}-x_{\alpha \delta}\right)\right\rangle, y \in S\left(x_{0}, r\right) .
$$

Using Taylor expression (see [20]) and taking $y=x_{\alpha \delta}$ we can write

$$
A^{\prime}\left(x_{0}\right)\left(x_{0}-x_{\alpha \delta}\right)-\frac{1}{2} A^{\prime \prime}\left(x_{0}\right)\left(x_{0}-x_{\alpha \delta}\right)^{2}=A\left(x_{0}\right)-A\left(x_{\alpha \delta}\right)+r_{\alpha \delta},\left\|r_{\alpha \delta}\right\| \leq L\left\|x_{\alpha \delta}-x_{0}\right\|^{3} / 6 .
$$

Therefore,

$$
\alpha 2^{2-\mu}\left\|x_{\alpha \delta}-x_{0}\right\|^{\mu} \leq \delta\left\|x_{\alpha \delta}-x_{0}\right\|+\alpha\left\|\omega\left(x_{\alpha \delta}-x_{0}\right)\right\|\left(\delta+\alpha\left\|x_{\alpha \delta}\right\|+L\left\|x_{\alpha \delta}-x_{0}\right\|^{3} / 6\right) .
$$

Hence

$$
\begin{gathered}
\left(2^{2-\mu}-\frac{L}{6}\left\|\omega\left(x_{\alpha \delta}-x_{0}\right)\right\|\left\|x_{\alpha \delta}-x_{0}\right\|^{3-\mu}\right)\left\|x_{\alpha \delta}-x_{0}\right\|^{\mu} \leq\left[\left(\delta+\alpha^{2}\left\|\omega\left(x_{\alpha \delta}-x_{0}\right)\right\|\right) / \alpha\right]\left\|x_{\alpha \delta}-x_{0}\right\| \\
+\left\|\omega\left(x_{\alpha \delta}-x_{0}\right)\right\|\left(\delta+\alpha\left\|x_{0}\right\|\right) .
\end{gathered}
$$

Since $x_{\alpha \delta} \rightarrow x_{0}$ and $\mu<3$, for sufficiently small $\alpha$ and $\delta$, we have

$$
\left.\left\|x_{\alpha \delta}-x_{0}\right\|^{\mu} \leq\left(\delta / \alpha+\alpha\left\|\omega\left(x_{\alpha \delta}-x_{0}\right)\right\|\right)+\left\|\omega\left(x_{\alpha \delta}-x_{0}\right)\right\|\left(\delta+\alpha\left\|x_{0}\right\|\right)\right) .
$$

Using the relation in [12]:

$$
a, b, c \geq 0, p>q, a^{p} \leq b a^{q}+c \Longrightarrow a^{p}=O\left(b^{p /(p-q)}+c\right)
$$

we obtain

$$
\left\|x_{\alpha \delta}-x_{0}\right\|=O\left(\delta^{q}\right), \quad q=\min ((1-p) /(\mu-1), p / \mu)
$$

Remark 1. If $A^{\prime \prime}\left(x_{0}\right)=0$ condition (iii) of Theorem will be written in the common form (iii) in Introduction with the righ-hand side $\partial \varphi\left(x_{0}\right)$. We shall see this in an example in Section 4. 
For numerical approximations one has to approximate the infinite dimensional Hilbert space $H$ by a sequence of finite-dimensional subspaces $H_{n}$ :

$$
H_{1} \subset H_{2} \subset \ldots \subset H_{n} \ldots \subset H, P_{n} x \rightarrow x, n \rightarrow+\infty, \forall x \in H,
$$

where $P_{n}$ denotes the orthogonal projections from $H$ onto $H_{n}$. Now, in place of (1.4), consider the finite-dimensional problems

$$
A_{n}(x)+\alpha \partial \varphi_{n}(x)=f_{\delta}^{n}, x \in H_{n}
$$

where $A_{n}=P_{n}^{*} A P_{n}, \partial \varphi_{n}=P_{n}^{*} \partial \varphi P_{n}$ and $f_{\delta}^{n}=P_{n}^{*} f_{b}$. It is easy to verify that $A_{n}$ and $\partial \varphi_{n}$ are monotone and continuous in $H_{n}$. Hence Eq. (2.2) has a unique solution $x_{\alpha \delta}^{n}$ for $\alpha>0$ and, for arbitrary $\alpha>0$ and $f_{\delta} \in H$, the sequence $\left\{x_{\alpha \delta}^{n}\right\}$ converges to $x_{\alpha \delta}$, as $n \rightarrow \infty$ (see [17]).

Theorem 2.2. Let the following conditions hold:

(i) $A$ is twice-Fréchet differentiable at some neighbourhood $U_{0}$ of $S_{0}$,

(ii) there exists a constant $L>0$ such that

$$
\left\|A^{\prime \prime}(x)-A^{\prime \prime}(y) \leq L\right\| x-y \|, x, y \in U_{0}
$$

and

(iii) $\alpha=\alpha(n, \delta)$ is such that $\alpha, \delta / \alpha \rightarrow 0$ and

$$
\left(\gamma_{n}(x)\left\|\left(I-P_{n}\right) x\right\|+L\left\|\left(I-P_{n}\right) x\right\|^{3} / 6\right) / \alpha \rightarrow 0, \forall x \in S_{0}
$$

as $n \rightarrow \infty$, where $\gamma_{n}(x)$ is denoted by

$$
\gamma_{n}(x)=\max \left\{\left\|A^{\prime}(x)\left(I-P_{n}\right)\right\|,\left\|A^{\prime \prime}(x)\left(I-P_{n}\right)\right\|\|x\|\right\},
$$

$I$ is the identity operator in $H$. Then the sequence $\left\{x_{\alpha \dot{ }}^{n}\right\}$ converges to $x_{0}$.

Proof. From (2.2) we have

$$
\begin{aligned}
\left\langle A_{n}\left(x_{\alpha \delta}^{n}\right)-A_{n}\left(x^{n}\right)\right. & \left.+\alpha\left(\partial \varphi_{n}\left(x_{\alpha \delta}^{n}\right)-\partial \varphi_{n}\left(x^{n}\right)\right), x_{\alpha \delta}^{n}-x^{n}\right\rangle=\left\langle f_{\delta}^{n}-A_{n}\left(x^{n}\right), x_{\alpha \delta}^{n}-x^{n}\right\rangle \\
& +\alpha\left\langle\partial \varphi_{n}\left(x^{n}\right), x^{n}-x_{\alpha \delta}^{n}\right\rangle, x^{n}=P_{n} x, x \in S_{0} .
\end{aligned}
$$

As

$$
\left.A\left(P_{n} x\right)=A(x)+A^{\prime}(x)\left(P_{n} x-x\right)+\frac{1}{2} A^{\prime \prime}(x)\left(P_{n} x-x\right)^{2}+r^{n},\left\|r^{n}\right\| \leq L \|\left(I-P_{n}\right) x\right) \|^{3} / 6, x \in S_{0},
$$


from (2.3) it implies

$$
\begin{gathered}
2^{2-\mu}\left\|x_{\alpha \delta}^{n}-x^{n}\right\|^{\mu} \leq\left(\delta+1.5 \gamma_{n}(x)\left\|\left(I-P_{n}\right) x\right\|+L\left\|\left(I-P_{n}\right) x\right\|^{3} / 6\right)\left\|x_{\alpha \delta}^{n}-x^{n}\right\| / \alpha \\
+\left\langle\partial \varphi\left(x^{n}\right), x^{n}-x_{\alpha \delta}^{n}\right\rangle .
\end{gathered}
$$

Consequently, the sequence $\left\{x_{\alpha \delta}^{n}\right\}$ is bounded. Let $x_{\alpha \delta}^{n}-x_{1}$ as $\alpha, \delta / \alpha \rightarrow 0$ and $n \rightarrow \infty$. Then $A_{n}\left(x_{\alpha \delta}^{n}\right) \rightarrow f_{0}$ follows from (2.2). We write the monotone property for $A_{n}$ :

$$
\left\langle A_{n}\left(x_{\alpha \delta}^{n}\right)-A_{n}\left(x^{n}\right), x_{\alpha \delta}^{n}-x^{n}\right\rangle \geq 0, \forall x \in H, x^{n}=P_{n} x .
$$

Therefore,

$$
\left\langle A_{n}\left(x_{\alpha \delta}^{n}\right)-A\left(x^{n}\right), x_{\alpha \delta}^{n}-x^{n}\right\rangle \geq 0 .
$$

From the last inequality and the continuity of $A$ it follows

$$
\left\langle f_{0}-A(x), x_{1}-x\right\rangle \geq 0, \forall x \in H,
$$

i.e. $x_{1} \in S_{0}$. Replacing $x^{n}$ by $x_{1}^{n}\left(=P_{n} x_{1}\right)$ in (2.4) we can conclude that the sequence $\left\{x_{\alpha \delta}^{n}\right\}$ converges strongly to $x_{1}$ and

$$
\begin{gathered}
2^{2-\mu}\left\|x_{\alpha \delta}^{n}-x^{n}\right\|^{\mu} \leq\left(\delta+1.5 \gamma_{n}(x)\left\|\left(I-P_{n}\right) x\right\|+L\left\|\left(I-P_{n}\right) x\right\|^{3} / 6\right)\left\|x_{\alpha \delta}^{n}-x^{n}\right\| / \alpha \\
+\left\langle\partial \varphi\left(x^{n}\right), x^{n}-x_{\alpha \delta}^{n}\right\rangle .
\end{gathered}
$$

After passing $\alpha, \delta \rightarrow 0$ and $n \rightarrow \infty$ in the last inequality we obtain

$$
\left\langle\partial \varphi(x), x-x_{1}\right\rangle \geq 0, \forall x \in S_{0}
$$

The last variational inequality is equivalent to $\left\langle\partial \varphi\left(x_{1}\right), x-x_{1}\right\rangle \geq 0, \forall x \in S_{0}$. Then $x_{1}=x_{0}$ and the entire sequence $\left\{x_{\alpha \delta}^{n}\right\}$ converges strongly to $x_{0}$.

$\otimes$.

Remark 2. From the above froof we can see that Theorem is still true if condition (iii) is replaced by

$$
\left.\gamma_{n}^{1}(x) / \alpha \rightarrow 0, \gamma_{n}^{1}(x)=\|\left(I-P_{n}\right) x\right) \|, x \in S_{0}
$$

We prove the following theorem in this case.

Theorem 2.3. Assume that the following conditions hold:

(i) conditions (i) - (iii) of Theorem 2.1 with $\omega(y) \equiv \omega_{1}, \forall y \in S\left(x_{0}, r\right)$ and (iii*) 
(ii) there exist two constants $L^{\prime}>0, \gamma^{\prime}>0$ such that

$$
\left\langle\partial \varphi(y)-\partial \varphi\left(x_{0}\right), z\right\rangle \leq L^{\prime}\left\|y-x_{0}\right\|^{\gamma^{\prime}}\|z\|, \forall y, z \in S\left(x_{0}, r\right)
$$

If we choose $\alpha=0\left(\left(\delta+\gamma_{n}^{1}\right)^{p}\right), 0<p<1$, and denote

$$
\gamma_{n}^{1}=\max \left\{\left\|\left(I-P_{n}\right) x_{0}\right\|,\left\|\left(I-P_{n}\right) \omega_{1}\right\|,\left\|\left(I-P_{n}\right) f_{0}\right\|\right\}
$$

Then

$$
\left\|x_{\alpha \delta}^{n}-x_{0}\right\|=O\left(\delta^{q_{1}}+\left(\gamma_{n}^{1}\right)^{q_{2}}\right), q_{1}=\min \{(1-p) /(\mu-1), p / \mu\} \text { and } q_{2}=\min \left\{\gamma^{\prime} /(\mu-1), q_{1}\right\}
$$

Proof. From (2.3) (with $x=x_{0}$ ),

$$
\left\|A\left(P_{n} x_{0}\right)-f_{\delta}\right\| \leq \delta+1.5 \tilde{\gamma}_{0}\left\|\left(I-P_{n}\right) x_{0}\right\|+L\left\|\left(I-P_{n}\right) x_{0}\right\|^{3} / 6
$$

where

$$
\tilde{\gamma}_{0}=\max \left\{\left\|A^{\prime}\left(x_{0}\right)\right\|,\left\|A^{\prime \prime}\left(x_{0}\right)\right\|\right\},
$$

and the monotonicity of $A_{n}$ it follows

$$
\begin{gathered}
\alpha 2^{2-\mu}\left\|x_{\alpha \delta}^{n}-x_{0}^{n}\right\|^{\mu} \leq \alpha\left\langle\partial \varphi\left(x_{0}^{n}\right), x_{0}^{n}-x_{\alpha \delta}^{n}\right\rangle+\left\|f_{\delta}-A\left(x_{0}^{n}\right)\right\|\left\|x_{\alpha \delta}^{n}-x_{0}^{n}\right\| \\
\leq\left(\delta+\tilde{\gamma}_{0} \gamma_{n}^{1}+\left(\gamma_{n}^{1}\right)^{3}\right)\left\|x_{\alpha \delta}^{n}-x_{0}^{n}\right\|+\alpha\left\langle\partial \varphi\left(x_{0}\right), x_{0}^{n}-x_{\alpha \delta}^{n}\right\rangle+\alpha\left\langle\partial \varphi\left(x_{0}^{n}\right)-\partial \varphi\left(x_{0}\right), x_{0}^{n}-x_{\alpha \delta}^{n}\right\rangle \\
\leq\left(\delta+\tilde{\gamma}_{0} \gamma_{n}^{1}+\left(\gamma_{n}^{1}\right)^{3}+\alpha L^{\prime}\left(\gamma_{n}^{1}\right)^{\gamma^{\prime}}\right)\left\|x_{\alpha \delta}^{n}-x_{0}^{n}\right\|+\alpha\left\langle\omega_{1}, A\left(x_{0}\right)-A\left(x_{\alpha \delta}^{n}\right)\right\rangle+\alpha\left\|\omega_{1}\right\|\left\|r_{\alpha \delta}^{n}\right\|,
\end{gathered}
$$

where

$$
\begin{gathered}
r_{\alpha \delta}^{n}=A\left(x_{\alpha \delta}^{n}\right)-A\left(x_{0}\right)+A^{\prime}\left(x_{0}\right)\left(x_{0}-x_{\alpha \delta}^{n}\right)-\frac{1}{2} A^{\prime \prime}\left(x_{0}\right)\left(x_{\alpha \delta}^{n}-x_{0}\right)^{2}, \\
\left\|r_{\alpha \delta}^{n}\right\| \leq L\left\|x_{\alpha \delta}^{n}-x_{0}\right\|^{3} / 6 \leq L\left\|x_{\alpha \delta}^{n}-x_{0}^{n}\right\|^{3} / 6+O\left(\gamma_{n}^{1}\right)
\end{gathered}
$$

and

$$
\left\langle\omega_{1}, A\left(x_{0}\right)-A\left(x_{\alpha \delta}^{n}\right)\right\rangle \leq\left\|\omega_{1}\right\|\left(\delta+\gamma_{n}^{1}+\left\|f_{\delta}^{n}-A_{n}\left(x_{\alpha \delta}^{n}\right)\right\|\right)+\left\langle\left(P_{n}-I\right) \omega_{1}, A\left(x_{\alpha \delta}^{n}\right)\right\rangle .
$$

Because of locally bounded property of every hemicontinuous and monotone operator (see $[20])$

$$
\left\langle\omega_{1}, A\left(x_{0}\right)-A\left(x_{\alpha \delta}^{n}\right)\right\rangle \leq\left\|\omega_{1}\right\|\left(\delta+\gamma_{n}^{1}+C_{1} \alpha+C_{2}\left\|\left(I-P_{n}\right) \omega_{1}\right\|, C_{1}, C_{2}>0\right.
$$


Consequently

$$
\begin{gathered}
\alpha\left(2^{2-\mu}-\frac{L\left\|\omega_{1}\right\|}{6}\left\|x_{\alpha \delta}^{n}-x_{0}^{n}\right\|^{3-\mu}\right)\left\|x_{\alpha \delta}^{n}-x_{0}^{n}\right\|^{\mu} \leq\left(\delta+\tilde{\gamma}_{0} \gamma_{n}^{1}+\left(\gamma_{n}^{1}\right)^{3}+\alpha L^{\prime}\left(\gamma_{n}^{1}\right)^{\gamma^{\prime}}\right) \\
\left\|x_{\alpha \delta}^{n}-x_{0}^{n}\right\|+\alpha\left(\left\|\omega_{1}\right\|\left(\left(1+C_{2}\right) \gamma_{n}^{1}+\delta+C_{1} \alpha\right) .\right.
\end{gathered}
$$

Using, again, the relation in [12] we have

$$
\left\|x_{\alpha \delta}^{n}-x_{0}^{n}\right\|=O\left(\delta^{q_{1}}+\left(\gamma_{n}^{1}\right)^{q_{2}}\right)
$$

and

$$
\left\|x_{\alpha \delta}^{n}-x_{0}\right\|=O\left(\delta^{q_{1}}+\left(\gamma_{n}^{1}\right)^{q_{2}}\right) \quad \otimes
$$

\section{ITERATIVE METHOD}

Now consider an iterative method to solve the equation

$$
F(x) \equiv A(x)+\partial \varphi(x)=f, f \in R(A+\partial \varphi)
$$

where $A$ and $\partial \varphi$ are defined as above. In the case $\partial \varphi=I$, the unique solution $\tilde{x}$ of (3.1) can be found by iterative methods in [2], [3] and [4] since in this cases $\mu=2$.

Let $x^{1}$ be an arbitrary element of $H$. The sequence of iterations $x^{k}$ is constructed by the formula

$$
x^{k+1}=x^{k}-\beta_{k}\left(F\left(x^{k}\right)-f\right) /\left\|F\left(x^{k}\right)-f\right\| .
$$

Theorem 3.1. If the real numbers $\beta_{k}$ satisfy the conditions

$$
1>\beta_{n}>0, \beta_{n} \searrow 0, \sum_{n=1}^{\infty} \beta_{n}=+\infty, \sum_{n=1}^{\infty} \beta_{n}^{2}<+\infty
$$

then the sequence $\left\{x^{k}\right\}$ converges to $\tilde{x}$, as $k \rightarrow+\infty$.

Proof. Put

$$
\lambda_{k}:=\left\|x^{k}-\tilde{x}\right\|^{2}
$$

It is easy to see that

$$
\lambda_{k+1} \leq \lambda_{k}+2\left\langle x^{k+1}-x^{k}, x^{k}-\tilde{x}\right\rangle+\left\|x^{k+1}-x^{k}\right\|^{2} .
$$


From this inequality, the uniformly monotone property of $F$ (that is caused by that one of $\partial \varphi)$ and (3.2) we get

$$
\lambda_{k+1} \leq \lambda_{k}-2^{3-\mu} \beta_{k} \lambda_{k}^{\mu / 2} /\left\|F\left(x^{k}\right)-f\right\|+\beta_{k}^{2} .
$$

Therefore, the sequence $\left\{\lambda_{k}\right\}$ is bounded. Consequently, the sequences $\left\{x^{k}\right\}$ and $\left\{F\left(x^{k}\right)\right\}$ are bounded, too. Hence there exist constants $G_{1}, G_{2}>0$ such that

$$
\lambda_{k} \leq G_{1} \text { and }\left\|F\left(x^{k}\right)-f\right\| \leq G_{2} .
$$

We can write (3.3) in the form

$$
\Delta_{k+1} \leq \Delta_{k}-\frac{2^{3-\mu} G_{1}^{\mu / 2-1}}{G_{2}} \beta_{k} \Delta_{k}^{\mu / 2}+\beta_{k}^{2} / G_{1}, \quad \Delta_{k}=\lambda_{k} / G_{1}
$$

Repeating the proof of Lemma 3 in [17] to the last inequality with $s_{k}=\alpha_{1} \Delta_{1}^{\mu / 2-1}+\ldots+$ $\alpha_{k} \Delta_{k}^{\mu / 2-1}$ we can conclude that the sequence $\left\{\Delta_{k}\right\}$ tends to zero, as $k \rightarrow+\infty$. Theorem is proved.

\section{APPLICATION}

We now apply the obtained results of the previous sections to study the singular integral equation in form (see [8])

$$
\int_{0}^{t}|s-t|^{-\lambda} x(s) d s+F(x(t))=f_{0}(t), 0<\lambda<1,
$$

where $f_{0}(t) \in L_{2}([0,1])$ and $F(t)$ satisfies the following conditions:

(i) $F(t)$ is a differentiable function,

(ii) $|F(t)| \leq c_{0}+c_{1}|t| ; c_{i}>0 ; F\left(t_{1}\right) \leq F\left(t_{2}\right), t_{1} \leq t_{2}$.

Let the operators $K$ and $F$ define by

$$
K y(t)=\int_{0}^{1} k(t, s) x(s) d s,(F x)(t)=F(x(t))
$$

where $k(t, s)=0$ if $t \geq s$ and $k(t, s)=(t-s)^{-\lambda}$ if $s<t$. Then $K$ and $F$ are the monotone operators in $H=L_{2}([0,1])$. In addition, suppose $F$ is compact, therefore (4.1) is an illposed problem, because $K$ also is compact (see [10]). It is easy to see that $A=K+F$ is monotone and Fréchet differentiable. In this case the condition (iii) of Theorem 2.1 is described by

$$
\left(K^{*}+F^{\prime *}\left(x_{0}\right)-\frac{1}{2} F^{\prime \prime *}\left(x_{0}\right) y\right) \omega=\partial \varphi\left(x_{0}\right)
$$


and if $F^{\prime \prime}$ is Lipschitz continuous $A^{\prime \prime}$ also is Lipschitz continuous. If $F^{\prime \prime}\left(x_{0}\right)=0$, for istance, $F$ is linear on the set of solutions of (4.1), then (4.2) has a simple form

$$
\left(K^{*}+F^{* *}\left(x_{0}\right)\right) \omega=\partial \varphi\left(x_{0}\right)
$$

And, in particular, if $F^{\prime}\left(x_{0}\right) \equiv 0$ that condition has a very simple form $K^{*} \omega=\partial \varphi\left(x_{0}\right)$.

Consider a concrete example, when

$+$

$$
F(t)= \begin{cases}t, & t \leq 0 \\ 0, & 0<t<1 \\ 2(t-1), & t \geq 1\end{cases}
$$

with $x_{0}(t)=\tilde{c}_{0} t^{\beta}, \beta>0$, where $\tilde{c}_{0}$ is a constant satisfying the condition

$$
\mu\left\|x_{0}\right\|_{L_{2}([0,1])}^{\mu-1} \tilde{c}_{0}(\beta+3 / 2)=\stackrel{\infty}{1} .
$$

Then

$$
f_{0}(t)=c_{0} \int_{0}^{t}|t-s|^{-\lambda} s^{\beta+1 / 2} d s, \partial \varphi\left(x_{0}\right)(t)=t^{\beta+1 / 2} .
$$

In this case, $\omega(t)=\Gamma(\beta+3 / 2) t^{\beta} / \Gamma(\beta+1)$ (see [7]).

Without loss of generality, consider $\tilde{x}_{0}(t)=x_{0}(t) / \tilde{c}_{0}$ and $\tilde{f}_{0}(t)=f_{0}(t) / \tilde{c}_{0}$ with $\lambda=\beta=$ 0.5 and $\mu=2.5$.

The values of $f_{\delta}(t)$ are chosen as perturbations of the values $\tilde{f}_{0}(t)$ according to

$$
f_{\delta}(t)=\tilde{f}_{0}(t)+\delta
$$

We compute the regularized solution $x_{\alpha \delta}^{n}$ for this problem using the iterative method (3.2) with error estimate 0.001 and approximating the Hilbert space $L_{2}[0,1]$ by the sequence of linear subspaces $H_{n}$, where

$$
\begin{gathered}
H_{n}=L\left\{\psi_{1}, \psi_{2}, \ldots, \psi_{n}\right\}, \\
\psi_{j}= \begin{cases}1, & t \in\left[t_{j-1}, t_{j}\right], \\
0, & t \notin\left[t_{j-1}, t_{j}\right], j=1, \ldots n .\end{cases}
\end{gathered}
$$

It is wellknow that

$$
\left\|\left(I-P_{n}\right) y_{0}\right\|=O\left(n^{-2}\right), \text { where } P_{n} y=\sum_{j=1}^{n} y\left(t_{j}\right) \psi_{j}(t) .
$$

Now we apply Theorem 2.3 for $\alpha(n)=O\left(n^{-1}\right)$ and $\delta=O\left(n^{-2}\right)$. We should obtain the convergence rates $e^{n}=\left\|\tilde{x}_{0}-x_{\alpha \delta}^{n}\right\|$. 
All numerical results are obtained with FORTRAN programs on an IBM 3031.

\begin{tabular}{|c|c|c|}
\hline $\mathrm{n}$ & \multicolumn{2}{|c|}{ Calc. Results } \\
\cline { 2 - 3 } points & $\alpha(n)$ & $e^{n}$ \\
\hline 15 & 0.007143 & 0.115264 \\
\hline 21 & 0.005000 & 0.097654 \\
\hline 33 & 0.003125 & 0.083582 \\
\hline 65 & 0.001563 & 0.067732 \\
\hline
\end{tabular}

\section{REFERENCES}

1. Alber J.I. \& Ryazantseva I.P., Regularization for nonlinear equations involving monotone operators, Zh. Vychish. Math. i Math. Fiz. 15, 1975, 1283-1289 (in Russian).

2. Alber J.I., Recurrent relations and variational inequalities, Dokl. Acad. Nauk SSSR 270, 1983, 11-16 (in Russian).

3. Bruck R. E., A strongly convergent iterative solution of $0 \in U(x)$ for a maximal monotone operator $U$ in Hilbert space, Math. Anal.and Appl. 48, 1974, 114-126.

4. Bruck R. E., An iterative solution of variational inequality for certain monotone operators in Hilbert spaces, Bull. AMS 81, 1975, 890-892.

5. Nguyen Buong, On nonlinear ill-posed problems, JJIAM, to appear.

6. Engl H.W., Kunish K. \& Neubauer A., Convergence rates for Tikhonov regularization of nonlinear ill-posed problems, Inverse Problems 5, 1988, 523-540.

7. Gerlach W. \& Wolfersdorf L.v., On Approximate Computation of the Values of the Normal Derivative of Solutions to Linear Partial Differential Equations of Second Order with Application to Abel's Integral Equation, ZAMM, Z. angew. Math. Mech. 66, 1986 1, 31-36.

8. Guseinov A.I. \& Mukhtarov H., Introduction to the theory of nonlinear singular integral equations, Nauka, Moskva 1980 (in Russian).

9. Kluge R., Gleichmaßig monotone Potentialoperatoren euf einigen Funk - tionalraumen, Math. Narch. 126, 1986, 301-309.

10. Krasnoselskii M.A., Topological methods in the theory of nonlinear integral equations, Nauka, Moscow 1958 (in Russian).

11. Natterer F., Error bounds for Tikhonov regularization in Hilbert scales, Applic. Analysis 18, 1984, 29-37.

12. Neubauer A., An a-posteriori parameter choice for Tikhonov regularization in Hilbert scales leading to optimal convergence rates, SIAM J. Numer. Math. 25, 1988, 1313-1326. 
13. Neubauer A., Tikhonov regularization for nonlinear ill-posed problems: Optimal convergence rates and finite-dimensional approximation, Inverse Problems 55, 1989, 541-577.

14. Neubauer A., Tikhonov regularization of Nonlinear Ill-posed Problems in Hibert Scales, Johannes Kepler Univ. 417, 1990, Sept.

15. Neubauer A. \& Scherzer O., Finite-dimensional approximation of Tikhonov regularization of nonlinear ill-posed problems, Numer. Funct. Analysis and Optim. 11, (1\&2) 1990, 85-89.

16. Perov A.I. \& Yurgelas V.V., On convergence for one iterative method, Zh. Vychish. Math. i Math. Fiz. SSSR 17, 1977, 859-870.

17. Ryazantseva I.P., On Galerkin's method solving equations with discontinuous monotone operators, Izvestia Vyschix Uchebnix Zav., ser. Math. SSSR 1978, 7, 68-72 (in Russian).

18. Sibony M., Sur l'approximation d'équation et inéquations aux derivées partielles nonlinéaires de type monotone, J. Math. Anal. Appl. 34, 1972, 502-564.

19. Tikhonov A.N. \& Arsenin V.I., Solutions of ill-posed problems, New-York, Wiley, 1977.

20. Vainberg M.M., Variational method and method of monotone operators, Nauka, Moskva 1972 (in Russian). 